\title{
Efectos del clima organizacional y los riesgos psicosociales sobre la felicidad en el trabajo
}

\author{
Effects of organizational climate and psychosocial risks on happiness at work \\ Francisco Javier Díaz Pincheira* y Moisés Esteban Carrasco Garcés
}

Universidad de Concepción, Chile

Recibido el 30 de junio de 2016; aceptado el 29 de septiembre de 2017

Disponible en Internet el 17 de septiembre de 2018

\begin{abstract}
Resumen
En este estudio se revisan factores de clima laboral y riesgos psicosociales y la manera como estos afectan a la felicidad organizacional. Para esto se midieron tres variables (felicidad, clima laboral y riesgos psicosociales) con sus respectivas dimensiones en una muestra de 107 trabajadores de una institución educativa de la ciudad de Los Ángeles, Chile. Se aplicaron tres escalas previamente validadas en el medio nacional y se clasificaron los niveles de felicidad en rangos altos y bajos, igualmente se aplicó modelo probit binomial para establecer las relaciones entre las variables de clima organizacional y riesgos psicosociales. Los principales resultados se asocian a que los altos niveles de felicidad organizacional se explican por trabajos con altos estándares de desempeño, en donde se refuerce positivamente al trabajador y exista flexibilidad con las necesidades familiares.
\end{abstract}

Códigos JEL: M10, M12, M19.

Palabras clave: Felicidad organizacional; clima laboral; riesgos psicosociales.

\footnotetext{
*Autor para correspondencia.

Correo electrónico: franciscodiaz@udec.cl (F.J. Díaz Pincheira)

La revisión por pares es responsabilidad de la Universidad Nacional Autónoma de México.
} 


\begin{abstract}
This study check factors of work climate and psychosocial risks, and how these affect the happiness at the organizational. For this, we measured three variables (happiness, work climate and psychosocial risks) with their respective dimensions in a sample of 107 workers in the area of education in the city of Los Angeles, Chile. We applied three scales previously validated in the national average and ranked the happiness levels in ranges high and low; also we applied a binomial probit model to establish the relationships between the variables of climate organizational and psychosocial risks. The main results describe that the high levels of organizational happiness are explained by jobs with high performance standards, where workers are positively reinforced and there is flexibility with family needs.
\end{abstract}

JEL Classification: M10, M12, M19

Keywords: Accruals; Organizational happiness; organizational climate; psychosocial risks.

\title{
Introducción
}

El desarrollo sostenible de la organización debe considerar los aspectos sociales, económicos y ecológicos, sin embargo, el aspecto psicosocial se ha descuidado con frecuencia en la investigación (Posada y Aguilar, 2012). En los últimos años la psicología positiva organizacional ha desarrollado nuevas variables que afectan el desarrollo de las personas dentro de la organización siendo la felicidad una de estas nuevas dimensiones de análisis. La felicidad por lo tanto es clave en la calidad de vida de las personas, ya sea en lo personal como en lo organizacional, facilitando la construcción de mejores relaciones interpersonales, aumentando la persistencia en el logro de objetivos, la productividad, la creatividad, la innovación, la satisfacción laboral y la motivación intrínseca al interior de las empresas (Fernández, 2015). Baker, Greenberg y Hemingway (2006) han señalado que la felicidad organizacional es un elemento estratégico de gestión del área de recursos humanos. En vista de esto se hace necesario desarrollar este constructo e identificar que elementos tanto a nivel personal como organizacional posibilitan la felicidad en el trabajo. Fisher (2010) por ejemplo señala que el concepto de felicidad organizacional incluye la satisfacción en el trabajo, pero es mucho más amplio, ya que considera estar involucrado con la organización y sus funciones. El objetivo de esta investigación es conocer que dimensiones del clima organizacional y que factores de riesgos psicosociales afectan la felicidad organizacional. Finalmente buscamos aumentar el conocimiento de los factores organizacionales que generan impacto sobre la felicidad en el trabajo.

\section{Revisión de literatura}

La felicidad ha sido definida como una experiencia interna positiva que incluye una emoción placentera, satisfacción con la vida, ausencia de emociones negativas, autorrealización y crecimiento personal (Andrews y Withey, 1976; Ryff, 1989). Moyano y Ramos (2007) sostienen que la felicidad es un estado emocional de las personas que parece estar alimentado cognitivamente por la reflexión acerca de su satisfacción general ante la vida y por la frecuencia de intensidad con que se experimentan emociones positivas. Históricamente se creyó que los países más felices eran aquellos que tenían un Producto Interno Bruto (PIB) más alto e ingresos per cápita mayores, sin embargo, varias investigaciones han descrito el 
efecto contrario, en donde una mejora objetiva de las condiciones materiales de la vida de las personas (salud, educación, economía) no traía necesariamente un avance en los niveles de felicidad (Veenhoven, 1994; Diener, 1984; Lyubomirsky 2007). Layard (2005) evidencia esto señalando una relación negativa entre aumento de riqueza y reducción de felicidad en el mundo más desarrollado, en donde el crecimiento económico no incrementa automáticamente la armonía social. Este fenómeno se ha replicado a nivel organizacional y la tendencia de la literatura actual sobre aspectos positivos de individuos en organizaciones se ha dirigido a la felicidad (Pashoal, Torres y Porto, 2010). La base de la felicidad en la organización son las emociones y los comportamientos organizacionales, es decir, la felicidad organizacional es el resultado de un pensamiento estratégico (Baker et al., 2006). Los mismos autores refieren que en organizaciones felices los colaboradores y directores están involucrados de forma efectiva y emocional con la organización, considerando el trabajo como una ocupación feliz, señalando que en las organizaciones felices los colaboradores tienen una actitud positiva y una alta motivación para laboral. Silverblatt (2010, citado por Dutschk, 2013) indica que los empleados no felices con su trabajo tienen un coste de millones en la economía, principalmente a través de la pérdida de productividad. El autor considera que promover la felicidad de los empleados es lo más importante y las emociones positivas suelen actuar como un antídoto frente a las emociones negativas, por lo que si el colaborador aprende a incrementar niveles de emoción positiva se siente menos estresado y más resistente. Fisher (2010) señala además que el concepto de felicidad organizacional considera dimensiones como la participación de los profesionales con la organización, satisfacción con el trabajo y compromiso positivo con la organización. Baker et al., (2006) en base en estudios de caso indican que en organizaciones felices los colaboradores son más creativos y capaces de provocar cambios e intentan transformar posibilidades en soluciones reales que contribuyan a la sostenibilidad de la organización, por su parte, los líderes motivan un entorno que fomenta la colaboración, cooperación y responsabilidad de innovar e donde incentivado el trabajo en equipo y el positivismo . Hosie, Sevastos y Cooper (2007) en un trabajo de investigación con 400 profesionales en Australia, encontraron correlaciones positivas entre el rendimiento de la organización y empleados más felices; el modelo aplicado considera dimensiones que evalúan la felicidad profesional en cuanto a características personales, características de las funciones laborales, definición de objetivos, flujo de trabajo, equilibrio entre trabajo y familia y satisfacción en el trabajo. Se ha señalado igualmente que la felicidad en el trabajo es posible asociarla con una mayor creatividad laboral y con un aumento de conductas prosociales con los compañeros de trabajo y los clientes, generando como resultado menores índices de conductas contraproductivas (Rodríguez y Sanz 2013). Dutschk (2013) en un estudio en Portugal logró identificar ocho factores de la felicidad organizacional mediante un análisis factorial los cuales son: desarrollo personal, reconocimiento y respeto, ambiente de trabajo, objetivos, equilibrio vida social, influencia y apoyo de jefes y compromiso con la organización y seguridad. La satisfacción laboral también se ha relacionado fuertemente con los niveles generales de la felicidad (Bowling, Eschleman, y Wang, 2010; Erdogan, Bauer, Trujillo, y Mansfield, 2012). En su meta-análisis de la satisfacción laboral y el bienestar subjetivo, Bowling et al., (2010) encontraron que la satisfacción en el trabajo se asoció positivamente con la satisfacción con la vida y la felicidad. Statuf, Monteiro, Pereira, Esgalhado Afonso y Loureiro (2016) en un estudio realizado en 971 personas en Portugal describieron que la satisfacción laboral estaba fuertemente correlacionada con la felicidad y la dimensión emocional de la salud; por su parte 
una alta satisfacción en el trabajo incrementa las probabilidades de reportar buenos niveles de energía, aumenta la calidad y la cantidad de interacciones sociales y proporciona a los trabajadores una protección adicional contra la ansiedad, la depresión y la pérdida de control emocional y conductual. Wright y Cropanzano (2004, citados por Moccia, 2016) mencionan que cuanto más alto es el nivel de felicidad y de emociones positivas de los trabajadores más fuerte es el vínculo entre la satisfacción en el trabajo, la ejecución y los resultados. La primera variable que analizamos fue la de clima laboral y sus efectos sobre la felicidad organizacional. El clima laboral u organizacional es definido como las percepciones compartidas de políticas, prácticas y procedimientos organizacionales, tanto formales como informales ( Reichers y Schneider, 1990). Así también puede que existan múltiples climas dentro de la misma organización, ya que la vida en la organización puede variar en cuanto a las percepciones de los miembros según los niveles de la misma, sus diferentes lugares de trabajo o las diversas unidades dentro del mismo centro de trabajo (Litwin y Stringer, 1968; Schneider y Hall, 1972). James y James (1989, Citados por Hernández, Méndez y Contreras, 2014) caracterizaron cinco dominios primarios de las percepciones sobre el ambiente de trabajo: 1) características del trabajo: autonomía, reto e importancia de la tarea 2) características del papel o rol laboral: ambigüedad, conflicto y sobrecarga 3) características del liderazgo: énfasis en las metas, apoyo e influencia ascendente 4) trabajo en equipo y características sociales del ambiente: cooperación, orgullo y calidez 5) atributos de la organización y el subsistema o departamento: innovación, apertura a la información y sistema de recompensas y reconocimientos. Por su parte Koys y Decottis (1991) incluyeron como dimensiones del clima organizacional: la autonomía, cohesión, equidad, presión, innovación, reconocimiento, confianza y apoyo. Parker, Baltes, Young, Huff, Altmann, Lacost, y Roberts (2003) a través de uno de los estudios más relevantes identificaron dimensiones comunes en las investigaciones del clima a nivel prácticamente mundial como: rol en el trabajo, el trabajo en sí, el líder, el grupo de trabajo, la organización en general, la satisfacción en el trabajo, el sentirse bien en el ambiente laboral, la motivación, el desempeño y otras actitudes hacia el trabajo. En la tabla $\mathrm{n}^{\mathrm{o}} 1$ se aprecian las dimensiones de clima laboral descritas por Koyts y Decottis (1991).

Tabla 1

Descripción de las ocho escalas de Clima Organizacional Koyts y Decottis, (1991)

\begin{tabular}{|c|c|}
\hline Autonomía & $\begin{array}{l}\text { Percepción del trabajador acerca de la autodeterminación y responsabilidad con respecto a } \\
\text { procedimientos laborales, metas y prioridades. }\end{array}$ \\
\hline Cohesión & Percepción de las relaciones interpersonales entre los trabajadores de la organización \\
\hline Equidad & $\begin{array}{l}\text { Percepción que los empleados tienen, acerca de sí existen políticas y reglamentos equitativos y } \\
\text { claros dentro de la institución. }\end{array}$ \\
\hline Presión & $\begin{array}{l}\text { Percepción que existe con respecto a los estándares de desempeño, funcionamiento y finaliza- } \\
\text { ción de la tarea }\end{array}$ \\
\hline Innovación & $\begin{array}{l}\text { Percepción que se tiene acerca del ánimo para asumir riesgos, ser creativos y adoptar nuevas } \\
\text { áreas de trabajo, donde se obtenga alguna experiencia }\end{array}$ \\
\hline Reconocimiento & $\begin{array}{l}\text { Percepción que tienen los miembros de la organización, con respecto a la recompensa que reci- } \\
\text { ben, como consecuencia al aporte positivo en el desarrollo de la organización }\end{array}$ \\
\hline Confianza & $\begin{array}{l}\text { Percepción de la libertad para comunicarse con los superiores, con el fin de tratar temas sensi- } \\
\text { bles y personales con la confidencia absoluta que genera seguridad que esa comunicación no } \\
\text { será violada o usada en contra de los miembros }\end{array}$ \\
\hline Apoyo & $\begin{array}{l}\text { Percepción que tienen los miembros acerca del respaldo y la tolerancia en el comportamiento } \\
\text { dentro de la institución para lo que se tendrá en cuenta el aprendizaje de los errores, por parte } \\
\text { del trabajador, sin temor a represalias por parte de sus superiores o compañeros de trabajo. }\end{array}$ \\
\hline
\end{tabular}

Fuente: Elaborado a partir de Koys y Decottis, (1991) 
Por su parte los riesgos psicosociales son definidos como situaciones laborales que tienen una alta probabilidad de dañar gravemente la salud de los trabajadores, física, social o mentalmente siendo escenarios que afectan habitualmente y de forma importante y grave la salud (Moreno, 2011). A partir del binomio trabajador- organización vs. productividad, Tous, Bonasa, Mayor y Espinosa (2011) exponen que los factores de riesgo psicosocial pueden afectar no solo el bienestar y la salud de los trabajadores sino que también influir de forma negativa en el rendimiento laboral. Los riesgos psicosociales se presentan en la tabla 2

Tabla 2

Dimensiones de riesgo psicosocial contenidas en el instrumento SUSESO-ISTAS21.

\begin{tabular}{|c|c|c|}
\hline Dimensiones & Sub- dimensiones & Descripción \\
\hline \multirow[t]{5}{*}{$\begin{array}{l}\text { Exigencias } \\
\text { Psicológicas }\end{array}$} & $\begin{array}{l}\text { Exigencias } \\
\text { Cuantitativas }\end{array}$ & $\begin{array}{l}\text { Cantidad o volumen de trabajo exigido contrastado con el tiempo disponi- } \\
\text { ble para realizarlo. }\end{array}$ \\
\hline & $\begin{array}{l}\text { Exigencias } \\
\text { Cognitivas }\end{array}$ & $\begin{array}{l}\text { Exigencias sobre diferentes procesos mentales (atención, memoria, decisio- } \\
\text { nes) y responsabilidad por las consecuencias de lo que se hace. }\end{array}$ \\
\hline & $\begin{array}{l}\text { Exigencias } \\
\text { emocionales }\end{array}$ & $\begin{array}{l}\text { Exigencias para no involucrarnos en la situación emocional que se deriva } \\
\text { de las relaciones interpersonales que implica el trabajo, especialmente } \\
\text { en aquellas ocupaciones en la que se prestan servicios a las personas y se } \\
\text { pretende inducir cambios en ellas }\end{array}$ \\
\hline & $\begin{array}{l}\text { Exigencias de } \\
\text { esconder emociones }\end{array}$ & $\begin{array}{l}\text { Demanda de ocultamiento de las emociones que surgen en el transcurso del } \\
\text { trabajo, normalmente ante la presencia de otras personas. }\end{array}$ \\
\hline & $\begin{array}{l}\text { Exigencias } \\
\text { sensoriales }\end{array}$ & $\begin{array}{l}\text { Exigencias laborales que significan utilizar los sentidos, en especial la } \\
\text { visión, con una alta atención y alerta a los detalles. }\end{array}$ \\
\hline \multirow[t]{5}{*}{$\begin{array}{l}\text { Trabajo activo } \\
\text { y desarrollo de } \\
\text { habilidades }\end{array}$} & Influencia del trabajo & $\begin{array}{l}\text { Margen de autonomía respecto al contenido y las condiciones de trabajo } \\
\text { (secuencia de la tarea, métodos a utilizar, tareas a realizar, cantidad de } \\
\text { trabajo, horarios, elección de compañeros). }\end{array}$ \\
\hline & $\begin{array}{l}\text { Posibilidades de } \\
\text { desarrollo }\end{array}$ & $\begin{array}{l}\text { Se evalúa si el trabajo es fuente de oportunidades de desarrollo de las } \\
\text { habilidades y conocimientos de cada persona. }\end{array}$ \\
\hline & $\begin{array}{l}\text { Control sobre el } \\
\text { tiempo de trabajo }\end{array}$ & $\begin{array}{l}\text { Posibilidad de pausar o interrumpir momentáneamente la tarea, sea para } \\
\text { un descanso breve, por atender obligaciones personales o para tomar } \\
\text { vacaciones. }\end{array}$ \\
\hline & Sentido del trabajo & Relacionar el trabajo con valores y objetivos trascendentales. \\
\hline & $\begin{array}{l}\text { Integración en la } \\
\text { empresa }\end{array}$ & $\begin{array}{l}\text { Identificación de cada persona con la empresa o institución en general. No } \\
\text { se refiere al contenido del trabajo en sí. }\end{array}$ \\
\hline \multirow{5}{*}{$\begin{array}{l}\text { Apoyo social } \\
\text { en la empresa } \\
\text { y calidad del } \\
\text { liderazgo }\end{array}$} & Claridad del rol & $\begin{array}{l}\text { Grado de definición de las acciones y responsabilidades del puesto de } \\
\text { trabajo. }\end{array}$ \\
\hline & Conflicto del rol & $\begin{array}{l}\text { Exigencias contradictorias que se presentan en el trabajo que pueden gene- } \\
\text { rar conflictos de carácter profesional o ético, cuando las exigencias de lo } \\
\text { que hay que hacer son diferentes de las normas y valores personales }\end{array}$ \\
\hline & Calidad de liderazgo & $\begin{array}{l}\text { Conductas y atributos del jefe o supervisor directo que permiten juzgar su } \\
\text { valor como líder. Incluye la capacidad de dirimir conflictos, planificar y } \\
\text { distribuir el trabajo en forma ecuánime, preocupación por el bienestar de } \\
\text { sus subordinados y habilidades de comunicación. }\end{array}$ \\
\hline & $\begin{array}{l}\text { Calidad de la } \\
\text { relación con } \\
\text { compañeros }\end{array}$ & $\begin{array}{l}\text { Relaciones con los compañeros de trabajo que se expresan tanto en formas } \\
\text { de comunicación como en la posibilidad de recibir el tipo de ayuda para } \\
\text { realizar el trabajo en el momento adecuado, así como el sentido de perte- } \\
\text { nencia a un equipo. }\end{array}$ \\
\hline & $\begin{array}{l}\text { Calidad de la } \\
\text { relación con sus } \\
\text { superiores }\end{array}$ & $\begin{array}{l}\text { Atributos tanto del jefe directo como de la organización en general que } \\
\text { posibilita recibir el tipo de ayuda e información que se necesita y en el } \\
\text { momento adecuado para realizar el trabajo. }\end{array}$ \\
\hline
\end{tabular}




\begin{tabular}{lll}
\cline { 2 - 3 } Compensaciones Estima & $\begin{array}{l}\text { Reconocimiento y apoyo de los superiores y compañeros por el esfuerzo } \\
\text { realizado para desempeñar el trabajo. Incluye recibir un trato justo }\end{array}$ \\
\cline { 2 - 3 } Doble Presencia & $\begin{array}{l}\text { Inseguridad respecto } \\
\text { a las condiciones del } \\
\text { contrato }\end{array}$ & $\begin{array}{l}\text { Preocupación por las condiciones del contrato, estabilidad o renovación, } \\
\text { variaciones del sueldo, formas de pago del sueldo, posibilidades de despido } \\
\text { y ascenso. }\end{array}$ \\
\cline { 2 - 3 } & $\begin{array}{l}\text { Inseguridad respecto } \\
\text { a las características } \\
\text { del trabajo que realiza }\end{array}$ & $\begin{array}{l}\text { Incluye la inseguridad sobre condiciones de trabajo tales como movilidad } \\
\text { funcional (cambios de tareas) y geográfica, cambios de la jornada y horario }\end{array}$ \\
\hline $\begin{array}{ll}\text { Preocupación de } \\
\text { tareas domesticas }\end{array}$ & $\begin{array}{l}\text { Intranquilidad provocada por las exigencias domesticas que puedan afectar } \\
\text { el desempeño laboral. }\end{array}$ \\
\hline
\end{tabular}

Fuente: Alvarado, Pérez Franco, Saavedra, Fuentealba, Alarcón, Marchetti, Aranda (2012)

Las investigaciones que buscan relacionar variables de clima laboral, riesgos psicosociales y felicidad organizacional son escazas, debido principalmente al estudio reciente de algunos de estos constructos. En cuanto a esto destaca la investigación llevada a cabo por Contreras, Juárez, Barbosa y Uribe (2010) quienes analizaron las relaciones entre los riesgos psicosociales, clima organizacional y el estilo de liderazgo en empresas colombianas del sector de servicios sociales y de salud en cuatro ciudades del país (Barranquilla, Bogotá, Cali y Bucaramanga). Este estudio muestra como el clima organizacional funcional reduce el estrés, favoreciendo el bienestar o felicidad, mientras que por el contrario los climas disyuntivos conducen a la presencia de riesgos en el trabajo. En cuanto a estudios que describan las relaciones entre los elementos de clima laboral con la felicidad organizacional se destaca lo realizado por George y Brief (1992) quienes evidencian los beneficios de la felicidad sobre el comportamiento organizacional, en donde los colaboradores con un nivel alto de felicidad son más cooperativos con sus compañeros, brindan sugerencias constructivas hacia el trabajo y están más comprometidos con su desarrollo profesional. Hay evidencia de que las personas más felices tienen resultados más favorables relacionados con el trabajo y que aquellos que regularmente experimentan emociones positivas y estados de ánimo agradables se sienten más seguros en la consecución de sus metas y son más propensos a enfrentarse eficazmente a desafíos (Statuf et al., 2016) Por su parte Warr (2013) ha estudiado los factores que generan felicidad e infelicidad en las organizaciones, el autor menciona que existen 12 factores (ver tabla 3 ) en las empresas que generaran felicidad y algunos de estos serían coincidentes con los elementos del clima organizacional. El autor realiza una analogía entre su investigación y el uso de las vitaminas en cuanto a que estas últimas son importantes para la salud física y a bajos niveles de consumo puede dar lugar a un deterioro fisiológico, sin embargo, una vez alcanzado un nivel moderado de vitaminas (cantidad diaria recomendada) no existe ningún beneficio a partir de cantidades adicionales, señala además que algunas vitaminas se convierten en perjudiciales en cantidades muy grandes por lo que la relación entre el aumento de la ingesta de vitaminas y la salud física se vuelve negativa después de un consumo constante de cantidades moderadas. En vista de esto señala que los primeros seis elementos se comportarían según esta última regla, en donde su exceso repercute directamente sobre la disminución de la felicidad en el trabajo mientras que la maximización de los últimos seis no afectaría en nada el desarrollo de la felicidad en el trabajo. 
Tabla 3

Principales características laborales que afectan a la felicidad o infelicidad

\begin{tabular}{|c|c|c|}
\hline A1 & Oportunidad de control & $\begin{array}{l}\text { Autonomía, libertad de decisión, participación, libertad en la toma de } \\
\text { decisiones }\end{array}$ \\
\hline A2 & $\begin{array}{l}\text { Oportunidad para el uso y la } \\
\text { adquisición de habilidades }\end{array}$ & $\begin{array}{l}\text { Entorno potencial para el uso y desarrollo de competencias y conoci- } \\
\text { mientos }\end{array}$ \\
\hline A3 & Metas generadas externamente & $\begin{array}{l}\text { Desafío, baja carga y sobrecarga de trabajo, identificación con la tarea, } \\
\text { conflicto de rol, trabajo emocional, conflicto trabajo-casa }\end{array}$ \\
\hline A4 & Variedad & Cambios en el contenido de las tareas y los contactos sociales. \\
\hline A5 & Claridad del entorno & $\begin{array}{l}\text { Resultados predecibles, requisitos claros, claridad de rol, retroalimen- } \\
\text { tación sobre las tareas, baja ambigüedad respecto al futuro. }\end{array}$ \\
\hline A6 & Contacto con otros & $\begin{array}{l}\text { Contacto social, calidad en las relaciones sociales, dependencia de los } \\
\text { demás, trabajo en equipo }\end{array}$ \\
\hline A7 & Disponibilidad de dinero & Ingresos disponibles, nivel salarial. \\
\hline A 8 & Seguridad Física & $\begin{array}{l}\text { Condiciones de trabajo adecuadas, grado de riesgos, calidad de los } \\
\text { equipos de trabajo }\end{array}$ \\
\hline A9 & Posición socialmente valorada. & $\begin{array}{l}\text { Importancia de la tarea o función, contribución a la sociedad, status en } \\
\text { grupos valorados }\end{array}$ \\
\hline A10 & Apoyo de supervisor & $\begin{array}{l}\text { Consideración por parte de los jefes, trato justo por parte del supervi- } \\
\text { sor, preocupación por el bienestar propio. }\end{array}$ \\
\hline A11 & Desarrollo de carrera & Seguridad laboral, oportunidades de promoción \\
\hline A12 & Equidad. & $\begin{array}{l}\text { Justicia dentro de la propia organización, equidad en las relaciones de } \\
\text { la organización con la sociedad }\end{array}$ \\
\hline
\end{tabular}

Fuente: Warr (2013).

Gamero (2013) en una muestra de trabajadores de Arequipa, Perú encontró relaciones positivas entre felicidad y variables como la compatibilidad puesto trabajador, autonomía, reconocimiento, claridad de rol, desarrollo de carrera y compromiso organizacional, de igual modo evidencio correlaciones positivas entre felicidad y satisfacción laboral señalando que las fluctuaciones de felicidad afectan más fuertemente la percepción de satisfacción laboral que lo que las fluctuaciones de satisfacción laboral afectan a la felicidad. En términos generales se podría sustentar que un individuo con un alto nivel de felicidad, bienestar psicológico, saludable y con control tanto de sí mismo (factor interno) como de su exterior (factor externo) podría sentirse bien consigo mismo, desarrollar relaciones positivas con los demás, moldear su medida para la satisfacción de necesidades, desarrollar la autonomía, encontrar un propósito para vivir, conservar el interés por la permanente búsqueda de crecimiento personal y del desarrollo de sus capacidades (Vásquez, Herbás, Ravona y Gómez, 2009). Entre algunas investigaciones que han establecido relaciones entre algunas dimensiones de los riesgos psicosociales y felicidad se destacan algunos estudios como los de Camargo, Fajardo y Correa (2012) quienes encontraron diversas asociaciones positivas referentes al dominio demandas del trabajo y bienestar del trabajador. Por su parte Aguilar, Rodríguez y Salanova (2002) analizaron la relación entre conductas de liderazgo del supervisor, riesgos psicosociales y felicidad en trabajadores españoles, en donde la muestra del estudio estuvo compuesta por 874 trabajadores de distintos sectores y categorías profesionales y se concluyó, que los resultados obtenidos en los análisis estadísticos del estudio muestran que el estilo de liderazgo que el supervisor emplee con sus colaboradores influirá de forma directa en el bienestar o felicidad que los trabajadores perciban, se evidenció además que los colaboradores que se encuentran más satisfechos son los que poseen líderes más centrados en los apoyos afectivos, Pozo, Salvador, Alonso y Martos (2008) en un estudio realizado en profesores determinaron que el 
apoyo social presenta una influencia directa en la felicidad disminuyendo los efectos negativos del desgaste en la salud. Finalmente, Arias, Masías y Justo (2014) estudiaron el efecto de las exigencias psicológicas asociado al burnout y su relación con el bienestar del trabajador encontrando relaciones negativas y significativas entre felicidad y agotamiento emocional, en donde los varones experimentan menores niveles de burnout que las mujeres, sin embargo las relaciones negativas entre felicidad y burnout son más fuertes en mujeres, de modo que la mujer más feliz experimenta menor agotamiento emocional.

\section{Materiales y métodos}

La población de estudio correspondió a una institución educativa de la ciudad de Los Ángeles, Chile (Liceo Alemán del Verbo Divino). No se estableció calculo muestral ya que se trabajó sobre el universo completo de la institución correspondiente a 107 trabajadores, en donde 33 individuos eran varones (30.8\%) y 74 mujeres. (69.2\%) con edades entre 24 y 64 años y una media de 46 años. Los instrumentos aplicados para investigación fueron tres: (a) La Escala de Felicidad Subjetiva (EFS) de Lyubomirsky y Lepper (1999) adaptado en Chile por Moyano y Ramos (2007) y que mide la felicidad global o duradera. Las personas responden un cuestionario en formato Likert con respuestas del 1 al 7, contestando reactivos tales como: "Algunas personas son muy felices en general, disfrutan de la vida independientemente de lo que suceda, sacan el máximo provecho del todo ¿Hasta qué punto Ud. es así?’. Para este instrumento en esta investigación se obtuvo una confiabilidad por consistencia interna mediante el coeficiente alfa de Cronbach de 0.64. (b) SUSESO ISTAS 21: Cuestionario para medir riesgos psicosociales en Chile siendo la traducción y validación del Copenhagen Psychosocial Questionnaire (COPSOQ). El cuestionario fue validado en Chile por la Superintendencia de Seguridad Social en colaboración con la Dirección del Trabajo, el Instituto de Salud Pública de Chile y la Escuela de Salud Pública de la Universidad de Chile. (Alvarado, Pérez-Franco, Saavedra, Fuentealba, Alarcón, Marchetti,y Aranda, 2012). Las personas responden un cuestionario en formato Likert igualmente con respuestas del 0 al 4. El cuestionario mide cinco variables: exigencias psicológicas, trabajo activo, apoyo social y liderazgo, compensaciones y doble presencia. Algunos ejemplos de reactivos son: "en su trabajo, ¿tiene usted que tomar decisiones difíciles?”, “en general, ¿considera usted que su trabajo le produce desgaste emocional?". Para este instrumento en esta investigación se obtuvo una confiabilidad por consistencia interna mediante el coeficiente alfa de Cronbach de 0.62. Por escala se obtuvieron los siguientes coeficientes: exigencias psicológicas 0.66; trabajo activo y desarrollo de habilidades 0.56; apoyo social en la empresa y calidad de liderazgo 0.70; compensaciones 0.64 y doble presencia 0.56. (c) Cuestionario de Clima Organizacional de Koyts y Decottis (1991). Este instrumento consta de 40 preguntas y las personas responden en una escala Likert de 1 a 5. El cuestionario mide ocho variables: autonomía, cohesión, confianza, presión, apoyo, reconocimiento, equidad e innovación. El instrumento fue adaptado en Chile por Chiang, Vega, Salazar y Nuñez (2008). Para este instrumento en esta investigación se obtuvo una confiabilidad por consistencia interna mediante el coeficiente alfa de Cronbach de 0.78. Por escala se obtuvieron los siguientes coeficientes: autonomía 0.83 ; cohesión 0.85 ; confianza 0.71 ; presión 0.72 ; apoyo 0.88 ; reconocimiento 0.81 ; equidad 0.50 e innovación 0.91 . Una vez recolectados los datos se organizaron y clasificaron de tal manera de posibilitar un mejor análisis de la información obtenida. Se realizó un análisis de fiabilidad del instrumento mediante el paquete estadístico SPSS 21.0, mientras que para lo obtención de los resultados econométricos se utilizó el paquete econométrico Stata 13.0 
Los modelos de elección discreta son apropiados cuando se deben modelar dos alternativas. Considere el uso típico del modelo, por ejemplo, para el caso de una empresa que debe decidir si realizar o no la compra de un insumo, o bien la contratación o no de un trabajador. En el caso del presente trabajo de investigación se busca saber los determinantes de que un trabajador tenga un nivel alto de felicidad en la organización. Un determinado trabajador tiene cierta propensión a sentirse feliz, $\boldsymbol{y}_{i}{ }^{*}$. Además, está linealmente relacionada con un vector de variables observables, $\boldsymbol{X}_{i}$, por ejemplo, variables relacionadas con el clima laboral y variables relacionadas con los riesgos psicosociales. Los factores que no podemos observar están considerados en el término de error, $\boldsymbol{\varepsilon}_{i}$ (ver ecuación (1))

$$
\boldsymbol{y}_{i}^{*}=\boldsymbol{\beta} \mathbf{X}_{i}+\varepsilon_{i}
$$

Cuando $\boldsymbol{y}_{i}^{*}$, es mayor a c

ierto umbral se considera que el trabajador tiene un nivel alto de felicidad. Por supuesto, no podemos observar la probabilidad de que un trabajador sea feliz, sólo se observa si este pasa el umbral, que llamaremos $\boldsymbol{y}_{i}$, y damos un valor de uno cuando el trabajador pasa el umbral y cero cuando no. La probabilidad de que $\boldsymbol{y}_{i}=1$, se modela por la ecuación (2), para el caso de un modelo probit binomial, donde $\boldsymbol{\beta}$ es el vector de coeficientes a estimar. Si los coeficientes son positivos significa que la probabilidad aumenta cuando la variable explicativa aumenta.

$$
\mathbf{P}\left(\boldsymbol{y}_{i}=1 \mid \mathbf{X}_{i}\right)=\boldsymbol{\Phi}\left(\mathbf{X}_{i}^{-} \beta\right)
$$

Donde $\boldsymbol{\Phi}$ es la función de densidad acumulada de la normal estándar.

Esta fórmula tiene dos características favorables. En primer lugar, está limitada entre 0 y 1, y es apropiada para una probabilidad. En segundo lugar, la distribución es intuitivamente atractiva. El impacto de los cambios en los coeficientes de la probabilidad de que ocurra un evento depende de la probabilidad inicial del evento.

\section{Resultados}

Relación entre felicidad y variables de clima laboral: Existen pocos estudios que relacionen estos factores, por lo que en esta investigación se intenta abordar dichas variables. Warr (2013), estudia algunos factores laborales que generan felicidad e infelicidad describiendo que en algunos casos si se llegan a niveles excesivos se podría generar infelicidad. Si observamos la tabla 4, en donde se muestran los efectos marginales del modelo de regresión probit para los niveles altos de felicidad, encontramos que la variable cohesión posee un efecto negativo sobre los niveles altos de felicidad (-0.133), es decir, aumentar la cohesión en una unidad disminuiría la probabilidad de encontrar niveles altos de felicidad en un $13 \%$ aproximadamente. Ahora bien, se podría pensar que para obtener niveles altos de felicidad se debe disminuir la cohesión o aislar a los trabajadores, en este sentido Warr (2013) menciona que la excesiva cohesión provocaría un desgaste en las relaciones humanas por exceder los límites básicos de la privacidad, en donde el trabajador entraría en una dinámica simbiótica con sus pares. 


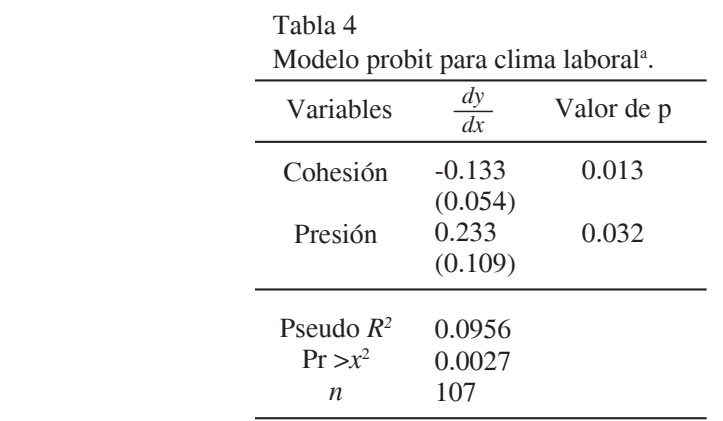

a Variable dependiente Felicidad (dicotómica). Desviación estándar entre paréntesis. Este modelo es un modelo depurado, se presentan los efectos marginales.

Ahora si observamos la variable presión esta muestra correlaciones positivas con la felicidad por lo que aumentar esta variable en una unidad incrementaría en un $23 \%$ la felicidad. Hay que entender que la variable presión se define como la percepción que existe con respecto a los estándares de desempeño, funcionamiento y finalización de la tarea. Es decir, un trabajador se mostrará feliz cuando existan metas claras y estándares altos de desempeño en el trabajo. Esto sería coincidente con lo señalado por Camargo, Fajardo y Correa (2012), quienes evidenciaron un resultado positivo de la presión sobre la felicidad del trabajador.

Por último, se observa la medida de bondad de ajuste (pseudo $R^{2}$ ) y la medida de significancia global $\left(\operatorname{Pr}>x^{2}\right)$. En primera instancia, si bien es bajo en nivel de bondad de ajuste (0.0956), debemos de considerar que el modelo intenta explicar la variabilidad de los altos niveles de felicidad en los trabajadores y ajustarse a esta variabilidad. La felicidad es una experiencia que influyen las emociones, es así, que lograr predecirlas es un alto desafío. No es erróneo considerar niveles bajos de ajuste cuanto intentamos predecir estos tipos de emociones. En segundo lugar, está la significancia global del modelo estimado (0.0027), esta nos muestra si las variables utilizadas para explicar los altos niveles de felicidad son significativas globalmente, se observa que a un nivel de $1 \%$ de significancia el modelo es significativo globalmente. En general, si bien el modelo presenta un bajo ajuste, las variables aquí presentadas son capaces de dar un poder explicativo a los altos niveles de felicidad.

Relación entre felicidad y dimensiones de riesgos psicosociales: En la literatura se sostiene que los riesgos psicosociales tienen una alta probabilidad de dañar la salud física, social y mental de los trabajadores (Moreno, 2011). En la tabla n ${ }^{\circ} 5$ se pueden apreciar las dimensiones de riesgos psicosociales que muestran una relación significativa con la felicidad.

En esta investigación la dimensión de exigencias psicológicas influye negativamente sobre los niveles altos de felicidad, en donde aumentar en una unidad las exigencias psicológicas provocaría un descenso del $19.8 \%$ de los niveles altos de felicidad, cabe mencionar que aumentar las exigencias psicológicas significa aumentar el volumen de trabajo en relación con el tiempo disponible para hacerlo, con una gran carga emocional. Ahora quisiéramos detenernos en este resultado, ya que si consideramos lo discutido en el apartado anterior la presión aumentaría la probabilidad de tener trabajadores altamente felices, pero si esta presión va acompañada de exigencias psicológicas los niveles de felicidad disminuirían. Esto coincide con lo señalado por Arias, Masías y Justo (2014), quienes encontraron una relación negativa entre felicidad y 
agotamiento emocional dado por trabajos emocionalmente desgastantes y que a la larga podrían generar altos niveles de burnout.

De igual modo se aprecia una relación negativa entre doble presencia y felicidad, es decir el aumento de una unidad de esta variable provocaría una disminución del 20.9\% de los niveles altos de felicidad, es decir, la intranquilidad provocada por las exigencias domesticas que puedan afectar el desempeño laboral influyen negativamente sobre la felicidad.

La variable compensaciones también muestra correlaciones negativas con la felicidad, en donde un aumento de una unidad de esta variable significa una disminución porcentual de la felicidad en un $16.1 \%$, es importante entender aquí que los puntajes altos en compensación se refieren a poco reconocimiento social en el trabajo, inseguridad respecto a las condiciones de trabajo e inseguridades con respecto al trabajo que se realiza.

Por último, al igual que en el caso de las variables de clima laboral, el modelo para los riesgos psicosociales presentan un bajo nivel de ajuste definidos por el Pseudo $R^{2}(0.1793)$, pero es un modelo significativo globalmente al $1 \%\left(\operatorname{Pr}>x^{2}=0.000\right)$.

Tabla 5

Modelo probit para Riesgos Psicosociales ${ }^{\mathrm{a}}$.

\begin{tabular}{l|l|c}
\hline Variables & $\frac{d y}{d x}$ & Valor de $\mathrm{p}$ \\
\hline Doble presencia & $\begin{array}{l}-0.209 \\
(0.075)\end{array}$ & 0.006 \\
Compensaciones & -0.161 & 0.041 \\
& $(0.079)$ & \\
Exigencias Psicológicas & -0.198 & 0.007 \\
\hline Pseudo $R^{2}$ & $(0.073)$ & \\
Pr $>x^{2}$ & \multicolumn{2}{|c}{0.1793} \\
$n$ & \multicolumn{2}{|c}{0.0000} \\
& \multicolumn{2}{|c}{107} \\
\hline
\end{tabular}

a Variable dependiente Felicidad (dicotómica). Desviación estándar entre paréntesis.

Este modelo es un modelo depurado, se presentan los efectos marginales.

\section{Conclusiones y discusión}

La escasa y reciente literatura encontrada que relaciona algunas dimensiones de riesgos psicosociales sobre la felicidad organizacional nos muestra que las dimensiones como la mala calidad del liderazgo, la poca claridad del rol y las exigencias psicológicas, afectan de manera negativa la felicidad en el trabajo (Aguilar, et. al., 2002; Pozo, et. al., 2008). Aquí a diferencia de estas investigaciones presentamos resultados que incluyen todas las dimensiones de los riesgos psicosociales sobre la felicidad. Es así que las dimensiones que resultaron ser significativas sobre la felicidad de los trabajadores fueron: la doble presencia, exigencias psicológicas y compensaciones. Todas estas dimensiones resultaron tener un efecto nocivo sobre la felicidad organizacional, resaltando el hecho de que las labores que poseen una gran carga y exigencia psicológica afectan la felicidad en el trabajo. De igual modo la variable doble presencia definida por Torns, Carrasquer y Borras (2002) como aquella realidad que se caracteriza por la combinación sincrónica y cotidiana del trabajo productivo y el doméstico aparece como una variable importante que afecta de manera negativa la felicidad, algunos autores como Fouche y 
Fartindale (2011) señalan que esta variable denominada igualmente conciliación trabajo familia debe equilibrarse para alcanzar la felicidad laboral y para esto se sugiere generar estrategias como la flexibilización laboral, la cual presenta un efecto benéfico y significativo sobre el contexto laboral y la disminución de los riesgos psicosociales (Recio, 1997). Boreham, Povey y Tomaszewski (2016) mencionan que las horas flexibles de trabajo son un componente común que permiten a los empleadores ayudar a cumplir con las presiones de carga de trabajo y retener a los empleados, por lo que los trabajos en donde exista poco reconocimiento de las labores, inseguridad contractual con respecto a las funciones generaran igualmente bajos niveles de felicidad.

Si consideramos la dimensión del clima laboral y la variable denominada presión está repercute positivamente sobre los niveles altos de felicidad organizacional, es necesario señalar que al hablar de presión nos referimos a altos estándares de desempeño y metas claras, es así que nuestros resultados nos hacen pensar que entregar labores con objetivos claros y metas a los trabajadores aumentarían la felicidad siempre y cuando estas labores no presenten una gran carga ni desgaste emocional. Un aspecto interesante es lo que ocurre con la variable cohesión, ya que nuestros resultados sugieren que aumentar está generaría menores niveles de felicidad; esto podría ser paradojico, pero Warr (2013) lo explica claramente señalando que existen ciertas variables del clima organizacional, una de ellas es la cohesión, que en exceso repercuten negativamente sobre la felicidad en el trabajo particularmente porque el contacto excesivo con otras personas en el trabajo puede afectar negativamente a la felicidad en situaciones de alta densidad o por medio de una falta de control personal, interrupciones frecuentes y el impedimento de llevar a cabo actividades interesantes debido a la demanda de los demás.

De esta investigación podemos señalar que la búsqueda de la felicidad es un deseo inherente de todos los seres humanos en todos los ámbitos de su vida, el trabajo por su parte también debiera ser una fuente de felicidad ya que pasamos gran parte de nuestro día en este. Csikszentmihalyi (2003) por ejemplo señala que el estado de flujo o florecimiento definido como: aquella experiencia en donde la persona se encuentra ensimismada en lo que está haciendo disfrutando de aquella actividad y perdiendo la noción del tiempo, solo se produce mientras los seres humanos realizan alguna actividad de trabajo, este tipo de experiencias son comunes por ejemplo entre los trabajadores que tienen mayor nivel de compromiso, involucramiento, motivación y satisfacción en su trabajo., por lo tanto las organizaciones debieran aprovechar esta situación para generar mayores instancias de felicidad en el trabajo

De igual modo investigaciones anteriores han descrito a la felicidad como un foco generador de satisfacción laboral, compromiso organizacional, capacidad innovadora, solución de problemas, mejora de relaciones interpersonales y productividad. En vista de esto sería necesario mantener activos y desarrollados a los trabajadores entregando metas y exigiendo estándares altos de desempeño, pero evitando las exigencias psicológicas. Es fundamental igual poder generar iniciativas que minimicen el conflicto trabajo- familia y flexibilicen las cargas horarias.

A nivel relacional hay que señalar que climas laborales cohesionados son importantes para desarrollar felicidad, sin embargo, si estos climas se vuelven invasivos y/o absorbentes podrían generar efectos adversos.

Estos resultados están sujetos a un tamaño muestral bajo, por lo que sería importante estudiar con una muestra mayor y con empresas de otros rubros, de igual modo sugerimos líneas de investigación asociadas a seguir estudiando los factores que afectan la felicidad organizacional y como esta se relaciona con la productividad del trabajador en la organización. 


\section{Referencias}

Aguilar, A; Rodríguez, A. y Salanova M. (2002) Estilos de liderazgo y riesgos psicosociales en los empleados. Jornadas de fomento de la investigación. Universidad Jaume I. Disponible en http://repositori.uji.es/xmlui/bitstream/ handle/10234/79722/Forum_2002_37.pdf?sequence=. Consultado el 20 de Marzo del 2016.

Alvarado, R., Pérez Franco, J., Saavedra, N., Fuentealba, C., Alarcón., Marchetti, N. y Aranda W. (2012). Validación de un cuestionario para evaluar riesgos psicosociales en el ambiente laboral en Chile. Revista Médica de Chile, 140 (1154-1163). https://doi.org/10.4067/s0034-98872012000900008

Andrews, F. y Withey, S. (1976). Social Indicators of Well-Being: Americans Perceptions of Life Quality. Nueva York: Plenum Press

Arias, W., Macías. A. y Justo O. (2014) Felicidad, burnout y estilos de afrontamiento en una empresa privada. Avances en psicología, 22(1) 75-88. Disponible en http://www.unife.edu.pe/publicaciones/revistas/psicologia/2014/ AVANCES.W.Arias.pdf. Consultado el 20 de enero del 2018.

Baker, D., Greenberg, C. y Hemingway, C. (2006). What Happy Companies Know, Pearson Education, New Jersey, USA.

Bowling, N., Eschleman, K. y Wang, Q. (2010). A meta-analytic examination of the relationship between job satisfaction and subjective well-being. Journal of Occupational and Organizational Psychology. 83(4), 915-934. https:// doi.org/10.1348/096317909x478557

Chiang, M., Salazar, M. y Nuñez, A (2008). Clima y satisfacción laboral en instituciones públicas. Adaptación y ampliación de un instrumento. Universum, 23 (2). 66-85. https://doi.org/10.4067/s0718-23762008000200004

Diener, E. (1984). Subjective Well Being: The Science of Happiness and a Proposal for a National Index. American Psychologist. 55(1), 34-43. https://doi.org/10.1037/0003-066x.55.1.34

Dutschk, G. (2013) Factores condicionantes de la felicidad organizacional en Portugal. Estudio exploratorio de la realidad en Portugal. Revista de Estudios Empresariales, 1, 21-43. Disponible en https://revistaselectronicas.ujaen.es/ index.php/REE/article/view/819/805. Consultado el 15 de junio del 2018.

Erdogan, B., Bauer, T., Truxillo, D. y Mansfield, L. R. (2012). Whistle while you work: A review of the life satisfaction literature. Journal of Management, 38(4), 1038-1083. https://doi.org/10.1177/0149206311429379

Fernandez, I. (2015). Felicidad Organizacional, como construir felicidad en el trabajo. Ediciones B. Chile

Fisher, C. (2010). Happiness at work. International Journal of Management Reviews. 12, 384-412. https://doi. org/10.1111/j.1468-2370.2009.00270.x

Fouché, C. y Martindale, K. (2011) Work-Life Balance: Practitioner Well-Being in the Social Work Education Curriculum. Social Work Education. 30-6, 675-685. https://doi.org/10.1080/02615479.2011.586566

Gamero, Y. (2013) La satisfacción laboral como dimensión de la felicidad. Ciencia \& Trabajo. 15(47) 94-102. https:// doi.org/10.4067/s0718-24492013000200010

George, J. y Brief, A. (1992). Feeling good-doing good: A conceptual analysis of the mood at work-organizational spontaneity relationship. Psychological Bulletin, 112(2), 310-329. https://doi.org/10.1037//0033-2909.112.2.310

Hernández, R, Méndez, S y Contreras, R. (2014) Construcción de un instrumento para medir el clima organizacional en función del modelo de los valores en competencia. Contaduría y Administración. 59(1) 229-257. https://doi. org/10.1016/s0186-1042(14)71250-1

Hosie, P. y Sevastos, P. (2009): Does the happy-productive worker thesis apply to managers?. International Journal of Workplace Health Management. 2 (2) 131-160. https://doi.org/10.1108/17538350910970219

Judge, T. y Watanabe, S. (1993). Another look at the job satisfaction-life satisfaction relationship. Journal of Applied Psychology, 78(6), 939-948. https://doi.org/10.1037/0021-9010.78.6.939

Koyts, D. y Decottis T. (1991) Inductive measures of psychologial climate. Human Relations. 44(3)265-385. https:// doi.org/10.1177/001872679104400304

Layard, R. (2005). La felicidad. Lecciones de una nueva ciencia, Taurus, México.

Lyubomirsky. S. (2007) La Ciencia De La Felicidad: Un Método Probado Para Conseguir el bienestar, Barcelona, Ediciones Urano.

Moccia, S. (2016) Felicidad en el trabajo. Papeles del Psicólogo. Revista del consejo general de psicología en España. 37(2). 143-151. Disponible en http://www.redalyc.org/pdf/778/77846055007.pdf. Consultado el 20 de marzo del 2018. 
Moyano, E. y Ramos, N. (2007) Bienestar Subjetivo: midiendo Satisfacción Vital, Felicidad y Salud en población chilena de la Región del Maule. Universum. 22 (2). 185-200. https://doi.org/10.4067/s0718-23762007000200012

Moreno, B. (2011) Factores y riesgos laborales psicosociales: conceptualización, historia y cambios actuales. Medicina y Seguridad del Trabajo. 57, 4-19. https://doi.org/10.4321/s0465-546x2011000500002

Parker, C; Baltes, B; Young, S; Huff, J; Altmann, R; Lacost, H. y Roberts, J. (2003) Relationships between psychological climate perceptions and work outcomes: meta-analytic review. Journal of Organizational Behavior, 24 (4) 389-416. https://doi.org/10.1002/job.198

Paschoal, T., Torres, C. y Porto, J. (2010): Felicidade no Trabalho: Relações com Suporte Organizacional e Suporte Social. Revista de Administração Comtemporânea. 14 (6), 1054-1072. https://doi.org/10.1590/s141565552010000700005

Posada, R. y Aguilar, O. (2012) La felicidad como elemento del desarrollo sostenible desde la organización. Revista del Centro de Investigación. Universidad La Salle, 10(37), 83-94

Pozo, C., Salvador, C., Alonso, E., \& Martos, M. (2008). Social support, burnout and well-being in teaching professionals. Contrast of a direct and buffer effect model. El apoyo social, el agotamiento y el bienestar de los profesionales de la enseñanza. Contraste de un modelo directo y el efecto amortiguador. Ansiedad y Estrés. 14(23), 127-141. Disponible en http://web.b.ebscohost.com/ehost/detail/detail?vid=0\&sid=7a8f817f-aab9-409f-a7a5 -d3a82af35aad\%40pdc-v-sessmgr02\&bdata=JnNpdGU9ZWhvc3QtbG12ZQ\%3d\%3d\#AN=40645150\&db=aph. Conusltado el 14 de febrero del 2018.

Recio, A. (1997). Trabajo, personas, mercados. Cuaderno de economía laboral. España: Icaria

Reichers, A. y Schneider, B. (1990). Climate and culture: An evolution of constructs. In B. Schneider (Ed.). Organizational Climate and Culture (5-39). San Francisco: Jossey - Bass.

Rodríguez, A y Sanz, A. (2013). Happiness and well-being at work: A special issue introduction. La felicidad y el bienestar en el trabajo: una introducción especial al tema. Revista de Psicología del Trabajo y de las Organizaciones. 29 (3), 95-97. https://doi.org/10.5093/tr2013a14

Ryff, C. (1989). Happiness is everything, or is it explorations on the meaning of psychological well-being. Journal of personality and social psychology. 57 (6) 1069- 1081. https://doi.org/10.1037//0022-3514.57.6.1069

Schneider, B. y Hall, D. (1972). Toward specifying the concept of work climate: A study of Roman Catholic diocesan priests. Journal of Applied Psychology. 56, 447-455. https://doi.org/10.1037/h0033756

Satuf, C.; Monteiro, S; Pereira, H.; Esgalhado, Alfonso R. y Loureiro, M. (2016) The protective effect of job satisfaction in health, happiness, well-being and self-esteem International Journal of Occupational Safety and Ergonomics. 1-9. https://doi.org/10.1080/10803548.2016.1216365

Tous, J., Bonasa, M., Mayor-Sanchez , C y Espinosa, I. (2011). Escala Clima Psicosocial en el Trabajo: desarrollo y validación. Anuario de Psicología. 41 (1-3), 51-65. Disponible en https://www.raco.cat/index.php/AnuarioPsicologia/article/viewFile/249848/334277. Consultado el 03 de enero del 2018.

Torns, T., Carrasquer, P. y Borrás, V. (2002). El estudio de la doble presencia: una apuesta por la conciliación de la vida laboral y familiar (informe de investigación) volumen 1. Barcelona: Universitat Autónoma de Barcelona.

Veenhoven, R. (1994). El estudio de la satisfacción con la vida. Intervención psicosocial. 3(9) 87-116. Disponible en https://personal.eur.nl/veenhoven/Pub1990s/94d-fulls.pdf. Consultado el 10 de diciembre del 2017.

Warr, P (2013) Fuentes de felicidad e infelicidad en el trabajo: una perspectiva combinada. Journal of Work and Organizational Psychology. 29 99-106. https://doi.org/10.5093/tr2013a15 